\title{
The differences in self-perceptions of aging, health-related quality of life and their association between urban and rural Chinese older hypertensive patients
}

\author{
Yunying Hou ${ }^{1,2+}$, Qing Wu ${ }^{1,2+}$, Dandan Zhang ${ }^{1}$, Xiaohong Jin ${ }^{3}$, Wenya Wu ${ }^{4}$ and Xiaohua Wang ${ }^{1,2^{*}}$ (D)
}

\begin{abstract}
Background: Most hypertensive clients are elderly, whose health-related quality of life (HRQL) may be associated with self-perceptions of aging (older individuals' beliefs about their own aging). Meanwhile, culture and health disparities between rural and urban populations are substantial. Whether there are differences in self-perceptions of aging, HRQL, and their association among elderly hypertensive clients in urban and rural areas remains unknown. The objective of this study was to investigate and compare self-perceptions of aging and HRQL and their association among urban and rural older Chinese hypertensive clients.
\end{abstract}

Methods: A cross-sectional investigation was conducted in 15 urban community clinics and 22 village clinics from Suzhou, China. Older hypertensive adults were invited to complete a self-administered questionnaire addressing sociodemographic and clinical information, HRQL and self-perceptions of aging.

Results: There were 492 urban participants and 537 rural participants included in the analyses. The physical (40.0 \pm 12.1 vs. $30.9 \pm 8.9, P<0.001)$ and mental (51.5 \pm 8.3 vs. $46.0 \pm 7.8, P<0.001) \mathrm{HRQL}$ scores of urban participants were all higher than those of rural ones. Urban participants' scores on dimensions of "timeline cyclical", "consequences negative", and "control negative" of self-perceptions of aging questionnaire (APQ) were lower than those of rural participants $(P<0.001$, respectively), while the scores on dimensions of "consequences positive" and "control positive" were higher $(P<0.001$, respectively). Adjusted multivariate linear regression showed that participants who had worse self-perceptions of aging had poorer HRQL. Some APQ dimensions associated with urban or rural hypertensive elders' HRQL were different.

Conclusions: Older hypertensive clients in rural areas have poorer self-perceptions of aging and HRQL than those in urban areas. Health care professionals should pay more attention to HRQL and self-perceptions of aging of older hypertensive clients in rural areas.

Keywords: Self-perceptions of aging, Health-related quality of life, Hypertension, Urban, Rural, Chinese

\footnotetext{
* Correspondence: sxwang2001@163.com

† Yunying Hou and Qing Wu contributed equally to this work.

'Department of Cardiology, the First Affiliated Hospital of Soochow

University, Suzhou 215006, China

${ }^{2}$ School of Nursing, Soochow University, Suzhou 215006, China

Full list of author information is available at the end of the article
}

(c) The Author(s). 2020 Open Access This article is licensed under a Creative Commons Attribution 4.0 International License, which permits use, sharing, adaptation, distribution and reproduction in any medium or format, as long as you give appropriate credit to the original author(s) and the source, provide a link to the Creative Commons licence, and indicate if changes were made. The images or other third party material in this article are included in the article's Creative Commons licence, unless indicated otherwise in a credit line to the material. If material is not included in the article's Creative Commons licence and your intended use is not permitted by statutory regulation or exceeds the permitted use, you will need to obtain permission directly from the copyright holder. To view a copy of this licence, visit http://creativecommons.org/licenses/by/4.0/. The Creative Commons Public Domain Dedication waiver (http://creativecommons.org/publicdomain/zero/1.0/) applies to the data made available in this article, unless otherwise stated in a credit line to the data. 


\section{Background}

The prevalence of hypertension is increasing [1], and most hypertensive patients are elderly. The prevalence of hypertension in older adults $\geq 65$ years in Mainland China is as high as $56.51 \%$ [2]. Hypertension is a major risk factor for coronary artery disease, stroke and renal failure, and it is one of the major causes of disability and death in the elderly [3]. Hypertensive older clients have more healthcare needs and are more likely to have poorer health-related quality of life (HRQL) than normotensive elderly people [4]. However, intensive treatment of blood pressure (BP) did not significantly affect HRQL [5]. Therefore, it is necessary to explore other factors associated with older hypertensive clients' poorer HRQL.

One of the factors that may be associated with quality of life is self-perceptions of aging [6,7]. Self-perceptions of aging were found to have a significant longitudinal impact on health and health behaviors in a meta-analysis of 19 studies [8]. Adults who have more positive selfperceptions of aging are more likely to engage in positive life style and have good psychological resources even in old age [9-13]. Older people who have the belief that health problems are inevitable in old age are unwilling to use preventive health service, and are more likely to have a heightened cardiovascular response to stress [14]. In contrast, poor health may lead to negative selfperceptions of aging $[15,16]$. Given the high prevalence of hypertension, and the possible positive change in selfperceptions of aging among older adults [17], promoting positive self-perceptions of aging may modify the poor HRQL of hypertensive older adults. However, the association between self-perceptions of aging and HRQL among older hypertensive people has not been thoroughly explored. Meanwhile, culture, thought and ideas, and health disparities between rural and urban populations have grown substantially with the process of rapid urbanization over past decades $[18,19]$. Whether there are differences in self-perceptions of aging, HRQL, and their association among elderly hypertensive clients in urban and rural areas remains unknown. The objective of the present study was to investigate and compare selfperceptions of aging and HRQL and their association among urban and rural older Chinese hypertensive clients.

\section{Methodology}

This study is a cross-sectional investigation, and information was gathered primarily through self-administered questionnaires, including basic socio-demographic and clinical data, self-reported HRQL, and self-perceptions of aging. Socio-demographic and clinical data included age, gender, marital status, educational attainments, living arrangements, status of BP control, duration of hypertension, the number of comorbidities and body mass index (BMI), which were covariates of HRQL and must be controlled when analyzing the association between self-perceptions of aging and HRQL [20]. After obtaining signed informed consent forms, investigators invited patients to complete the investigation. Nurse and student investigators were trained in questionnaire administration before the start of the survey, including data collection and verification methods, and how to communicate accurately and effectively with participants. For illiterate participants, investigator researchers read the question items word-by-word, exactly as printed on the questionnaire. At the end of the investigation, each participant had his or her BMI measured. This study was approved by the ethics committee of Changshu No.1 People's Hospital.

\section{Study population}

This cross-sectional survey was carried out in 15 urban community clinics and 22 rural village clinics in Suzhou, China. Because the population density of Suzhou municipality is less than 1500 people per square kilometer, we defined the areas where the sub-district offices were located and the constructed towns as urban, and the other areas where more than $50 \%$ residential population were farmers as rural, according to the demarcation standards of the National Bureau of Statistics and the State Council of China. There were approximately 3, 800 inhabitants in each urban community and 4, 800 inhabitants in each rural village. Participants aged 60 years or above who sought medical advice in study clinics were recruited between November 2013 and December 2016. Subjects who reported ever having been diagnosed with hypertension by a qualified health care provider and were able to communicate were invited to participate in this study. Hypertension was defined as systolic blood pressure $(\mathrm{SBP}) \geq 140$ $\mathrm{mmHg}$ and/or diastolic blood pressure (DBP) $\geq 90 \mathrm{mmHg}$ or the use of antihypertensive medications [21]. Each potential subject was verbally asked about the history of their diseases and medications and verified by their medical records to confirm study eligibility. Subjects were excluded if they had any of the following conditions that would prohibit participation or have a great impact on HRQL: dementia or cognitive impairment, cancer, heart failure (New York Heart Association functional class III or IV), or unstable angina.

We used G"Power version 3.1 software to estimate the sample size. With a medium effect size at 0.10 and a power of 0.90 , the sample size for 16 predictors to achieve $\alpha$ at 0.01 was 331. Considering that there might be $30 \%$ of questionnaires having missing or implausible information, the total sample size of urban and rural hypertensive patients should not be less than 860 . 


\section{Instrument}

The participants' age, gender, marital status, educational attainments, living arrangements, status of BP control, duration of hypertension, and the number of comorbidities were gathered through a self-reported survey by trained researchers. Hypertensive clients with $\mathrm{BP}<140$ / $90 \mathrm{mmHg}$ in their last appointment were considered to have their BP controlled. The height and weight were measured using a calibrated stadiometer and weight scale for patients wearing light clothing without shoes. The BMI was calculated as the weight (in kilograms) divided by the square of the height (in metres).

HRQL was measured using the short form-36 (SF-36), which has been used in different populations [22-24]. SF-36 is a self-administered questionnaire that generates assessment scores across 36 scales. These scales are scored on a $0-100$ scale, with higher scores indicating better HRQL. The following 8 dimensions of HRQL are evaluated by these scores: physical functioning (PF), role limitations due to physical problems (RP), bodily pain $(\mathrm{BP})$, general health perceptions $(\mathrm{GH})$, vitality $(\mathrm{VT})$, social functioning (SF), role limitations due to emotional problems (RE), and mental health $(\mathrm{MH})$. The SF-36 dimensions can be reduced to 2 aggregate summaries with the first four dimensions indicating respondents' physical component summary (PCS) and the last four indicating mental component summary (MCS) [25]. The mandarin version of the SF-36 used in this study has been administered successfully in general populations in China with the internal reliability coefficients of the PCS and MCS scales ranging from 0.85 to 0.87 [26, 27] and its validity and reliability have been tested in Chinese hypertensive clients [28]. The PCS and MCS scores were calculated based on the Chinese population norm [27].

Self-reported perceptions of aging were measured using the 32-item aging perceptions questionnaire (APQ) developed by Barker et al. in 2007 [29]. The 32 items comprised 7 subscales examining aging views: timeline chronic, timeline cyclical, consequences positive, consequences negative, control positive, control negative, and emotional representations. "Timeline chronic" relates to the awareness of the chronic nature of aging (e.g., 'I always classify myself as old'), and "timeline cyclical" reflects the variation in the awareness of aging (e.g., 'I go through phases of feeling old'). "Consequences" refers to beliefs about the impact of aging on one's life, including positive consequences (e.g., 'As I get older, I get wiser') and negative consequences (e.g., 'Getting older makes everything harder for me'). "Control" refers to elders' beliefs about managing one's experience of aging, which can be positive (e.g., 'The quality of my social life in later years depends on me') or negative (e.g., 'Immobility in later life is not up to me'). "Emotional representations" demonstrate the negative emotions generated by aging (e.g. 'I get depressed when I think about getting older'). Answers are provided on a 5point scale, ranging from 1 'strongly disagree' to 5 'strongly agree'. The response scale of the control negative dimension is reversed ( 1 'strongly agree' to 5 'strongly disagree'). The mean score for each subscale is calculated with higher scores indicating greater endorsement of a specific perception. A Chinese version of the APQ was used, with Cronbach alpha coefficients of 0.884 for the total scale, 0.869 for timeline chronic, 0.700 for timeline cyclical, 0.665 for consequences positive, 0.836 for consequences negative, 0.822 for control positive, 0.748 for control negative, 0.835 for emotional representations, and cumulative variance contribution rate $65.269 \%$ [30].

\section{Analytical strategy}

All data were analyzed using SPSS 20.0 (SPSS, Chicago, Illinois, USA). All continuous variables were expressed as mean \pm standard deviation (SD), and the categorical variables were summarized by frequencies or percentage. Socio-demographic and clinical characteristics were calculated for rural and urban participants, and the significance of differences across groupings were determined using the Chi-square test for nominal variables and Student's $t$-test for independent samples for continuous variables (Table 1). Multivariate analysis of variance (MANOVA) was used to control demographic characteristics and compare the differences of HRQL and selfperceptions of aging between urban and rural participants (Tables 2 and 3). Multivariate linear regression was utilized to assess the association between self-perceptions of aging and PCS and MCS adjusted for age (numerical), gender, marital status (single or divorced or widowed vs. married), educational attainments (junior middle school and below vs. senior high school and above), living arrangements (with family vs. alone), BMI (numerical), duration of hypertension (numerical), and comorbidities (with vs. without) (Tables 4 and 5). The coefficient and $95 \%$ confidence interval $(95 \% \mathrm{CI})$ are presented. All statistical tests were two-sided and tests of alpha less than 0.05 were considered statistically significant.

\section{Results}

\section{Participant characteristics}

A total of 1068 copies of the initial questionnaires were collected. Among those collected, 39 (3.7\%) were excluded due to missing data or implausible data, including persons who did not have a blood pressure measurement $(n=7)$ and those who did not complete the SF-36 HRQL or APQ or who chose the same score on each item when filling out APQ $(n=32)$. Finally, there were 492 urban participants and 537 rural participants included in the analyses. Table 1 presents the socio-demographic and clinical characteristics. Compared with participants who lived in rural areas, more 
Table 1 Socio-demographic and Clinical Characteristics for Urban and Rural Participants

\begin{tabular}{|c|c|c|c|c|}
\hline$\underline{\text { Variable }}$ & Urban participants $(n=492)$ & Rural participants $(n=537)$ & $x^{2} / t$ & $P$ \\
\hline Gender, $n$ (\%) & & & 4.890 & 0.027 \\
\hline Male & $263(53.5 \%)$ & $250(46.6 \%)$ & & \\
\hline Female & $229(46.5 \%)$ & $287(53.4 \%)$ & & \\
\hline Age (years), Mean $\pm S D$ & $68.4 \pm 7.5$ & $70.8 \pm 9.2$ & -4.569 & $<0.001$ \\
\hline Marital status, $n(\%)$ & & & 4.968 & 0.026 \\
\hline Single/divorced/widowed & $91(18.5 \%)$ & $130(24.2 \%)$ & & \\
\hline Married & $401(81.5 \%)$ & $407(75.8 \%)$ & & \\
\hline Educational attainments, $n$ (\%) & & & 196.371 & $<0.001$ \\
\hline Junior middle school and below & $316(64.2 \%)$ & $526(98.0 \%)$ & & \\
\hline Senior high school and above & $176(35.8 \%)$ & $11(2.0 \%)$ & & \\
\hline Living arrangements, $n(\%)$ & & & 3.342 & 0.068 \\
\hline Alone & $44(8.9 \%)$ & $32(6.0 \%)$ & & \\
\hline With family & $448(91.1 \%)$ & $505(94.0 \%)$ & & \\
\hline $\mathrm{BMI}\left(\mathrm{kg} / \mathrm{m}^{2}\right), M \pm S D$ & $23.6 \pm 3.2$ & $24.0 \pm 4.0$ & -1.845 & 0.065 \\
\hline Blood pressure control, $n$ (\%) & & & 158.759 & $<0.001$ \\
\hline Yes & $366(74.4 \%)$ & $189(35.2 \%)$ & & \\
\hline No & $126(25.6 \%)$ & $348(64.8 \%)$ & & \\
\hline Hypertension duration (years), Mean $\pm S D$ & $9.1 \pm 8.3$ & $10.8 \pm 7.2$ & -3.480 & 0.001 \\
\hline Comorbidities, n (\%) & & & 0.173 & 0.677 \\
\hline No & $263(53.5 \%)$ & $294(54.7 \%)$ & & \\
\hline Yes & $229(46.5 \%)$ & $243(45.3 \%)$ & & \\
\hline
\end{tabular}

Note. SD standard deviation, $B M I$ body mass index

participants living in urban areas were men $(53.5 \%$ vs. $46.6 \%, P=0.027)$, younger $(68.4 \pm 7.5$ vs. $70.8 \pm 9.2$ years old, $P<0.001)$, married ( $81.5 \%$ vs. $75.8 \%, P=0.026)$, had a higher education level (35.8\% vs. $2.0 \%$ had an education higher than senior high school, $P<0.001$ ) and shorter hypertension duration $(9.1 \pm 8.3$ vs. $10.8 \pm 7.2$ years, $P=0.001)$, and had their blood pressure under control (74.4\% vs. $35.2 \%, P<0.001)$.

\section{Urban and rural participants' HRQL and self-perceptions of aging}

Tables 2 and 3 present the results of urban and rural participants' self-rated HRQL and perceptions of aging. There were statistical differences in all of the dimension scales of HRQL between urban and rural participants except "general health" dimension. In total, urban participants had higher PCS $(40.0 \pm 12.1$ vs. $30.9 \pm 8.9, P<0.001)$ and MCS

Table 2 SF-36 Scores for Urban and Rural Participants

\begin{tabular}{|c|c|c|c|c|}
\hline Domain, Mean $\pm S D$ & Urban participants $(n=492)$ & Rural participants $(n=537)$ & $F$ & $P$ \\
\hline Physical functioning & $73.9 \pm 22.5$ & $70.5 \pm 23.9$ & 15.384 & $<0.001$ \\
\hline Role physical & $65.2 \pm 38.0$ & $80.2 \pm 36.7$ & 10.808 & 0.001 \\
\hline Bodily pain & $76.6 \pm 20.5$ & $9.1 \pm 13.0$ & $1.017 \mathrm{E} 3$ & $<0.001$ \\
\hline General health & $44.1 \pm 16.3$ & $45.8 \pm 6.2$ & 0.618 & 0.432 \\
\hline Vitality & $67.8 \pm 16.5$ & $59.6 \pm 14.8$ & 112.239 & $<0.001$ \\
\hline Social functioning & $85.4 \pm 16.4$ & $45.7 \pm 20.3$ & 285.632 & $<0.001$ \\
\hline Role emotional & $69.7 \pm 37.2$ & $85.4 \pm 31.8$ & 10.231 & 0.001 \\
\hline Mental health & $65.6 \pm 15.8$ & $57.9 \pm 14.8$ & 50.085 & $<0.001$ \\
\hline PCS & $40.0 \pm 12.1$ & $30.9 \pm 8.9$ & 144.300 & $<0.001$ \\
\hline MCS & $51.5 \pm 8.3$ & $46.0 \pm 7.8$ & 136.752 & $<0.001$ \\
\hline
\end{tabular}

Note. SD standard deviation, SF-36 36-item short-form health survey, PCS physical component summary, MCS mental component summary 
Table 3 Scores on Each Domain of Self-perceptions of Aging for Urban and Rural Participants

\begin{tabular}{|c|c|c|c|c|}
\hline Domain, Mean $\pm S D$ & Urban participants $(n=492)$ & Rural participants $(n=537)$ & $F$ & $P$ \\
\hline Timeline chronic & $3.0 \pm 0.9$ & $3.1 \pm 0.8$ & 0.017 & 0.897 \\
\hline Timeline cyclical & $3.3 \pm 0.7$ & $3.6 \pm 0.6$ & 32.923 & $<0.001$ \\
\hline Consequences positive & $3.5 \pm 0.7$ & $3.3 \pm 0.7$ & 17.493 & $<0.001$ \\
\hline Consequences negative & $3.1 \pm 0.8$ & $3.6 \pm 0.6$ & 114.586 & $<0.001$ \\
\hline Control positive & $3.6 \pm 0.7$ & $3.3 \pm 0.6$ & 45.438 & $<0.001$ \\
\hline Control negative & $3.0 \pm 0.7$ & $3.4 \pm 0.6$ & 19.993 & $<0.001$ \\
\hline Emotional representations & $2.7 \pm 0.8$ & $2.6 \pm 0.6$ & 0.428 & 0.513 \\
\hline
\end{tabular}

Note. SD standard deviation

$(51.5 \pm 8.3$ vs. $46.0 \pm 7.8, P<0.001)$ scores than rural participants. Urban participants' scores on dimensions of "timeline cyclical", "consequences negative", and "control negative" of self-perceptions of aging scale were lower than those of rural participants $(P<0.001$, respectively), while the scores on dimensions of "consequences positive" and "control positive" were higher $(P<0.001$, respectively).

\section{Association between self-perceptions of aging and HRQL}

The results of multivariate linear regression for the 7 dimensions scores of the self-perceptions of aging scale and the PCS and MCS scores adjusted for sociodemographic and clinical characteristics for urban and rural participants are presented in Tables 4 and 5 . Urban and rural participants who had higher scores on "emotional representations" dimension of selfperceptions of aging had lower PCS and MCS scores. Besides "emotional representations", for urban participants, "control positive" was the only dimension associated with both PCS and MCS; for rural participants, "timeline chronic" was the only dimension associated with both PCS and MCS. Urban participants' PCS was also associated with "consequences negative".

\section{Discussion}

Urban and rural participants' HRQL

By comparing with general Chinese older people reported in the literature [31], hypertensive older clients in this study had poor HRQL, and the rural participants had poorer physical and mental HRQL than those residing in urban settings. This finding agrees with the study

Table 4 Association between Self-perceptions of Aging and PCS for Urban and Rural Participants

\begin{tabular}{|c|c|c|c|c|c|c|}
\hline \multirow[b]{2}{*}{ Variable } & \multicolumn{3}{|c|}{ Urban participants $(n=492)$} & \multicolumn{3}{|c|}{ Rural participants $(n=537)$} \\
\hline & Coefficient & $95 \% \mathrm{Cl}$ & $P$ & Coefficient & $95 \% \mathrm{Cl}$ & $P$ \\
\hline \multicolumn{7}{|l|}{ Self-perceptions of aging } \\
\hline Timeline chronic & -1.17 & $-2.28,-0.06$ & 0.038 & -3.21 & $-4.24,-2.18$ & $<0.001$ \\
\hline Timeline cyclical & -0.01 & $-1.66,1.64$ & 0.988 & -1.91 & $-3.64,-0.18$ & 0.031 \\
\hline Consequences positive & -0.19 & $-1.54,1.16$ & 0.779 & 1.15 & $0.07,2.24$ & 0.037 \\
\hline Consequences negative & -2.07 & $-3.43,-0.71$ & 0.003 & 1.57 & $-0.21,3.35$ & 0.084 \\
\hline Control positive & 2.08 & $0.70,3.45$ & 0.003 & 0.34 & $-0.92,1.60$ & 0.596 \\
\hline Control negative & 0.26 & $-1.20,1.72$ & 0.724 & -1.45 & $-3.03,0.13$ & 0.073 \\
\hline Emotional representations & -1.50 & $-2.97,-0.03$ & 0.045 & -4.31 & $-5.56,-3.05$ & $<0.001$ \\
\hline \multicolumn{7}{|l|}{ Controlled variables } \\
\hline Gender (ref: male) & -2.36 & $-4.22,-0.49$ & 0.013 & -1.81 & $-3.16,-0.46$ & 0.009 \\
\hline Age & -0.20 & $-0.35,-0.06$ & 0.006 & -0.07 & $-0.17,0.03$ & 0.152 \\
\hline Marital status (ref: single/divorced/widowed) & 2.61 & $-0.17,5.38$ & 0.065 & 0.43 & $-1.35,2.22$ & 0.663 \\
\hline Educational attainments (ref: junior middle school and below) & -0.53 & $-2.49,1.43$ & 0.598 & -4.43 & $-9.16,0.29$ & 0.066 \\
\hline Living arrangements (ref: alone) & -2.15 & $-5.78,1.48$ & 0.246 & -1.84 & $-4.89,1.21$ & 0.236 \\
\hline BMl & -0.04 & $-0.32,0.24$ & 0.789 & 0.10 & $-0.07,0.27$ & 0.254 \\
\hline Blood pressure control (ref: no) & 0.68 & $-1.38,2.73$ & 0.519 & 0.16 & $-1.26,1.56$ & 0.830 \\
\hline Hypertension duration & -0.10 & $-0.22,0.02$ & 0.111 & -0.09 & $-0.20,0.01$ & 0.069 \\
\hline Comorbidities (ref: no) & -7.34 & $-9.35,-5.33$ & $<0.001$ & -0.15 & $-1.60,1.29$ & 0.834 \\
\hline
\end{tabular}


Table 5 Association between Self-perceptions of Aging and MCS for Urban and Rural Participants

\begin{tabular}{|c|c|c|c|c|c|c|}
\hline \multirow[b]{2}{*}{ Variable } & \multicolumn{3}{|c|}{ Urban participants $(n=492)$} & \multicolumn{3}{|c|}{ Rural participants $(n=537)$} \\
\hline & Coefficient & $95 \% \mathrm{Cl}$ & $P$ & Coefficient & $95 \% \mathrm{Cl}$ & $P$ \\
\hline \multicolumn{7}{|l|}{ Self-perceptions of aging } \\
\hline Timeline chronic & 0.11 & $-0.75,0.97$ & 0.801 & -2.52 & $-3.47,-1.56$ & $<0.001$ \\
\hline Timeline cyclical & -0.86 & $-2.14,0.43$ & 0.191 & 0.17 & $-1.45,1.78$ & 0.841 \\
\hline Consequences positive & 0.45 & $-0.60,1.50$ & 0.399 & -0.25 & $-1.25,0.76$ & 0.632 \\
\hline Consequences negative & -0.04 & $-1.10,1.01$ & 0.935 & -1.13 & $-2.79,0.52$ & 0.179 \\
\hline Control positive & 1.89 & $0.82,2.96$ & 0.001 & 0.62 & $-0.55,1.78$ & 0.302 \\
\hline Control negative & 0.94 & $-0.20,2.07$ & 0.106 & 1.43 & $-0.04,2.91$ & 0.057 \\
\hline Emotional representations & -2.41 & $-3.55,-1.26$ & $<0.001$ & -1.72 & $-2.88,-0.55$ & 0.004 \\
\hline \multicolumn{7}{|l|}{ Controlled variables } \\
\hline Gender (ref: male) & 1.32 & $-0.13,2.77$ & 0.074 & 1.57 & $0.31,2.83$ & 0.015 \\
\hline Age & 0.20 & $0.09,0.31$ & 0.001 & -0.02 & $-0.12,0.07$ & 0.632 \\
\hline Marital status (ref: single/divorced/widowed) & -0.42 & $-2.58,1.73$ & 0.701 & -1.64 & $-3.30,0.02$ & 0.053 \\
\hline Educational attainments (ref: junior middle school and below) & 0.49 & $-1.03,2.02$ & 0.526 & -1.11 & $-5.51,3.28$ & 0.620 \\
\hline Living arrangements (ref: alone) & 1.19 & $-1.63,4.02$ & 0.407 & 1.18 & $-1.66,4.01$ & 0.416 \\
\hline BMI & 0.18 & $-0.04,0.40$ & 0.101 & -0.10 & $-0.26,0.06$ & 0.210 \\
\hline Blood pressure control (ref: no) & 0.26 & $-1.34,1.86$ & 0.752 & -0.22 & $-1.54,1.09$ & 0.738 \\
\hline Hypertension duration & 0.04 & $-0.06,0.13$ & 0.435 & 0.05 & $-0.05,0.14$ & 0.331 \\
\hline Comorbidities (ref: no) & -2.65 & $-4.22,-1.09$ & 0.001 & 0.51 & $-0.84,1.85$ & 0.458 \\
\hline
\end{tabular}

Note. $\mathrm{Cl}$ confidence interval, MCS mental component summary, BMI body mass index

conducted in Shanxi Province, China, which found that urban hypertensive adults had higher HRQL than those in rural areas [32]. More physical/structural and services/assistance barriers [33], and worse economic conditions experienced by rural population may contribute to this difference.

\section{Urban and rural participants' self-perceptions of aging}

The present study found that older hypertensive rural residents expressed more negative self-perceptions of aging. This is consistent with the study by $\mathrm{Li} \mathrm{X}$ et al. [13] who found that the elderly who lived in rural areas had lower expectations regarding aging than those in urban areas. Differences in culture and ethical values between rural and urban areas caused by disparities in economic and educational levels may account for the discrepancy of aging perceptions. This is in accordance with the study conducted in Southern Italy, which found that social, environmental, and economic imparities between metropolitan and rural areas determined the difference in aging perceptions among elders aged 75 years or more [34].

The association between self-perceptions of aging and HRQL among urban and rural participants

Multivariate regression analysis showed that the dimension of "emotional representations" of APQ was associated with both urban and rural participants' physical and mental HRQL. Individuals who had strong negative emotional responses to aging were inclined to functional disability and depression, resulting in late life maladaptive outcomes [35, 36].

The findings of this study are fairly consistent with those of previous studies demonstrating quality of life as a function of self-perceptions of aging [10, 13]. Possible mediating roles of self-perceptions of aging in the relationship between a poor health condition and quality of life have also been suggested in some studies [6, 37]. Self-perceptions of aging were suggested to be a mediator or moderator of relationships between the subjective health of older adults and quality of life in 20 countries [6] and those between comorbidity and quality of life of older home care clients [37].

\section{The differences in the association between self- perceptions of aging and HRQL among urban and rural participants}

Multivariate regression analysis showed that aging perceptions dimensions which were associated with urban or rural participants' HRQL were different. For urban hypertensive elders, besides "emotional representations", "control positive" was the more prominent one which was associated with both physical and mental HRQL, with more positive control indicating better HRQL. According to reports in the literature, older hypertensive patients who had a positive sense of control over their 
health tended to control hypertension through their own efforts, such as lifestyle changes [10], diet modification [38], as well as medication adherence, which has been demonstrated among urban older hypertensive clients in our previous study [39]. Urban participants' physical HRQL was also associated with "consequences negative". High "consequences negative" perception may cause older adults lose confidence in their abilities, resulting in a gradual conscious or unconscious withdrawal in engagement from activities [40]. Different from urban hypertensive elders, rural participants' physical and mental HRQL were negatively associated with "timeline chronic" dimension. Chronic timeline perception makes older adults always classify themselves as old, tend to explain physical problems in terms of an age attribution, rather than an extenuating circumstance, thus delaying or unwilling to seek treatment for health problems [41].

\section{Clinical relevance}

The findings of this study imply a possibility that the promotion of positive self-perceptions of aging will improve the HRQL of older hypertensive patients. Given the results of our study showing that the dimensions of self-perceptions of aging associated with rural or urban older hypertensive patients' physical or mental HRQL were different, targeted intervention programs could be designed for rural and urban hypertensive elders to improve their physical and mental HRQL. These interventions may include providing information about positive aspects in old age and false stereotypes about old adults, finding arguments against the negative view on aging, and strengthening the positive view on aging through opinion expression, group discussion, quiz, homework and other techniques based on cognitive behavioral therapy principles. Some researchers have confirmed that the implementation of a self-perceptions of aging intervention could improve mental health of the elderly [42]. Considering the poorer aging perceptions and HRQL, and the larger population of rural elders [43], more measures are warranted among these people.

\section{Limitations}

There are some limitations to this study. First, the sampling was not stratified randomized; therefore, the participants in the study may not adequately represent older hypertensive clients. Moreover, given this study took place in limited regions of China, generalizability of the findings to other countries cannot be guaranteed. Second, the relationship between self-perceptions of aging and HRQL was estimated according to a crosssectional design; it is difficult to establish the causal association even controlling for the confounders. Third, although the total scale of the Chinese version APQ used in this study had acceptable internal consistency reliability, the reliability of the "emotional representations" subscale was low, with the Cronbach alpha coefficient less than 0.7 .

\section{Conclusions}

HRQL was related to self-perceptions of aging in Chinese older hypertensive clients. Rural or urban older adults' HRQL was associated with different self-perceptions of aging dimensions. Health care professionals should not only control older patients' blood pressure but also pay attention to their HRQL and self-perceptions of aging, and develop targeted intervention programs for rural and urban older hypertensive people to improve their HRQL.

\section{Abbreviations}

HRQL: Health-related quality of life; BP: Blood pressure; BMI: Body mass index; SBP: Systolic blood pressure; DBP: Diastolic blood pressure; PF: Physical functioning; RP: Role limitations due to physical problems; BP: Bodily pain; GH: General health perceptions; VT: Vitality; SF: Social functioning; RE: Role limitations due to emotional problems; MH: Mental health; PCS: Physical component summary; MCS: Mental component summary; APQ: Aging perceptions questionnaire; SD: Standard deviation; Cl: Confidence interval

\section{Acknowledgements}

We thank the older adults with hypertension who volunteered to participate in this study. Thanks also to all the staff in studied urban community and rural village clinics in Suzhou.

\section{Authors' contributions}

Study concept and design: XW, YH, QW. Acquisition of data: DZ, WW Analysis and interpretation of data: $\mathrm{YH}, \mathrm{QW}, \mathrm{XW}$. Drafting of the manuscript: YH, QW. Revision of the manuscript: XW. The author(s) read and approved the final manuscript.

\section{Funding}

No funding was received for this study.

Availability of data and materials

Please contact corresponding author for data requests.

\section{Ethics approval and consent to participate}

This study was approved by the ethics committee of Changshu No.1 People's Hospital. Participants read and singed informed consent agreements prior to the initiation of the study.

\section{Consent for publication}

Not applicable.

\section{Competing interests}

The authors declare no conflicts of interest.

\section{Author details}

'Department of Cardiology, the First Affiliated Hospital of Soochow University, Suzhou 215006, China. ${ }^{2}$ School of Nursing, Soochow University, Suzhou 215006, China. ${ }^{3}$ Quality Improvement Office, Changshu No.1 People's Hospital, Changshu 215500, China. ${ }^{4}$ Department of Cardiology, Changshu No.1 People's Hospital, Changshu 215500, China.

Received: 16 September 2018 Accepted: 19 May 2020 Published online: 26 May 2020

\section{References}

1. Yang Z, Li W, Tu X, Tang W, Messing S, Duan L, Pan J, Li X, Wan C. Validation and psychometric properties of Chinese version of SF-36 in patients with hypertension, coronary heart diseases, chronic gastritis and peptic ulcer. Int J Clin Pract. 2012;66(10):991-8. 
2. Gao Y, Chen G, Tian H, Lin L, Lu J, Weng J, Jia W. Prevalence of hypertension in China: a cross-sectional study. PLoS One. 2013;8(6):e65938.

3. Rosendorff C, Lackland D, Allison M, Aronow W, Black H, Blumenthal R, Cannon C. Treatment of hypertension in patients with coronary artery disease: a scientific statement from the American Heart Association, American College of Cardiology, and American Society of Hypertension. Hypertension. 2015;65(6):1372-407.

4. Saleem F, Hassali MA, Shafie AA. A cross-sectional assessment of healthrelated quality of life (HRQoL) among hypertensive patients in Pakistan. Health Expect. 2014;17(3):388-95.

5. O'Connor PJ, Narayan KM, Anderson R, Feeney P, Fine L, Ali MK, Simmons DL, Hire DG, Sperl-Hillen JM, Katz LA, et al. Effect of intensive versus standard blood pressure control on depression and health-related quality of life in type 2 diabetes: the ACCORD trial. Diabetes Care. 2012;35(7):1479-81.

6. Low G, Molzahn AE, Schopflocher D. Attitudes to aging mediate the relationship between older peoples' subjective health and quality of life in 20 countries. Health Qual Life Outcomes. 2013;11:146.

7. Low G, Molzahn A, Kalfoss M. Cultural frames, qualities of life, and the aging self. West J Nurs Res. 2014:36(5):643-63.

8. Westerhof GJ, Miche M, Brothers AF, Barrett AE, Diehl M, Montepare JM, Wahl HW, Wurm S. The influence of subjective aging on health and longevity: a meta-analysis of longitudinal data. Psychol Aging. 2014;29(4): 793-802.

9. Andrews RM, Tan EJ, Varma VR, Rebok GW, Romani WA, Seeman TE, Gruenewald TL, Tanner EK, Carlson MC. Positive Aging Expectations Are Associated With Physical Activity Among Urban-Dwelling Older Adults. Gerontologist. 2017;57(suppl_2):S178-86.

10. Breda Al, Watts AS. Expectations regarding aging, physical activity, and physical function in older adults. Gerontol Geriatr Med. 2017;3:602186802.

11. Kim ES, Moored KD, Giasson HL, Smith J. Satisfaction with aging and use of preventive health services. Prev Med. 2014;69:176-80.

12. Korkmaz AG, Kartal A, Ozen Cl, Kostu N. The relationship between attitudes toward aging and health-promoting behaviours in older adults. Int J Nurs Pract. 2017;23(6):e12594.

13. Li X, LV Q, Li C, Zhang H, Li C, Jin J. The relationship between expectation regarding aging and functional health status among older adults in China. J NursScholarsh. 2013;45(4):328-35.

14. Levy BR, Ryall AL, Pilver CE, Sheridan PL, Wei JY, Hausdorff JM. Influence of African American elders' age stereotypes on their cardiovascular response to stress. Anxiety Stress Coping. 2008;21(1):85-93.

15. Harrison T, Blozis S, Stuifbergen A. Longitudinal predictors of attitudes toward aging among women with multiple sclerosis. Psychol Aging. 2008; 23(4):823-32.

16. Rozanova J, Noulas P, Southwick SM, Pietrzak RH. Perceptions of determinants of successful aging among older U.S. veterans: results from the National Health and resilience in veterans study. Am J Geriatr Psychiatry. 2015:23(7):744-53.

17. Brothers A, Diehl M. Feasibility and efficacy of the aging (plus) program: changing views on aging to increase physical activity. J Aging Phys Act. 2017;25(3):402-11.

18. Miao J, Wu X. Urbanization, socioeconomic status and health disparity in China. Health Place. 2016;42:87-95.

19. Poel EV, O'Donnell O, Doorslaer EV. Is there a health penalty of China's rapid urbanization? Health Econ. 2012;21(4):367-85.

20. Xu X, Rao Y, Shi Z, Liu L, Chen C, Zhao Y. Hypertension impact on healthrelated quality of life: a cross-sectional survey among middle-aged adults in Chongqing, China. Int J Hypertens. 2016;2016:7404957.

21. Chobanian AV, Bakris GL, Black HR, Cushman WC, Green LA, Izzo JJ, Jones DW, Materson BJ, Oparil S, Wright JJ, et al. Seventh report of the joint National Committee on prevention, detection, evaluation, and treatment of high blood pressure. Hypertension. 2003;42(6):1206-52.

22. Brazier JE, Harper R, Jones NM, O'Cathain A, Thomas KJ, Usherwood T, Westlake L. Validating the SF-36 health survey questionnaire: new outcome measure for primary care. BMJ. 1992;305(6846):160-4.

23. Ware JJ. SF-36 health survey update. Spine (Phila Pa 1976). 2000;25(24): 3130-9.

24. Jenkinson C, Coulter A, Wright L. Short form 36 (SF36) health survey questionnaire: normative data for adults of working age. BMJ. 1993; 306(6890):1437-40

25. Taft C, Karlsson J, Sullivan M. Do SF-36 summary component scores accurately summarize subscale scores? Qual Life Res. 2001;10(5):395-404
26. Li L, Wang HM, Shen Y. Chinese SF-36 health survey: translation, cultural adaptation, validation, and normalisation. J Epidemiol Community Health. 2003;57(4):259-63.

27. Lam CL, Tse EY, Gandek B, Fong DY. The SF-36 summary scales were valid, reliable, and equivalent in a Chinese population. J Clin Epidemiol. 2005; 58(8):815-22.

28. Wang R, Zhao Y, He X, Ma X, Yan X, Sun Y, Liu W, Gu Z, Zhao J, He J. Impact of hypertension on health-related quality of life in a population-based study in Shanghai, China. Public Health. 2009;123(8):534-9.

29. Barker M, O'Hanlon A, McGee HM, Hickey A, Conroy RM. Cross-sectional validation of the aging perceptions questionnaire: a multidimensional instrument for assessing self-perceptions of aging. BMC Geriatr. 2007;7:9.

30. Chen X, Hu Y, Zhu D, Li J, Zhou L. Chinese version of the aging perceptions questionnaire (C-APQ): assessment of reliability and validity. Aging Ment Health. 2016:20(6):567-74.

31. Song T, Ding Y, Sun Y, He Y, Qi D, Wu Y, Wu B, Lang L, Yu K, Zhao X, et al. A population-based study on health-related quality of life among urban community residents in Shenyang, Northeast of China. BMC Public Health 2015;15:921.

32. Zhang Y, Zhou Z, Gao J, Wang D, Zhang Q, Zhou Z, Su M, Li D. Healthrelated quality of life and its influencing factors for patients with hypertension: evidence from the urban and rural areas of Shaanxi Province, China. BMC Health Serv Res. 2016;16:277.

33. Cao Y, Walker EA, Krause JS. Environmental barriers and subjective health among people with chronic spinal cord injury: a cohort study. J Spinal Cord Med. 2015:38(4):526-31.

34. Lucchetti M, Corsonello A, Gattaceca R. Environmental and social determinants of aging perception in metropolitan and rural areas of southern Italy. Arch Gerontol Geriatr. 2008;46(3):349-57.

35. Robertson DA, Savva GM, King-Kallimanis BL, Kenny RA. Negative perceptions of aging and decline in walking speed: a self-fulfilling prophecy. PLoS One. 2015;10(4):e123260.

36. Kaori K, Richard Z, Clyde BS, Nir B, Gil A. Positive attitude towards life, emotional expression, self-rated health, and depressive symptoms among centenarians and near-centenarians. Aging Ment Health. 2016;20(9):930-9.

37. Yamada Y, Merz L, Kisvetrova H. Quality of life and comorbidity among older home care clients: role of positive attitudes toward aging. Qual Life Res. 2015;24(7):1661-7.

38. Fukuoka Y, Lindgren TG, Bonnet $K$, Kamitani E. Perception and sense of control over eating behaviors among a diverse sample of adults at risk for type 2 diabetes. Diabetes Educ. 2014;40(3):308-18.

39. Hou Y, Zhang D, Gu J, Xue F, Sun Y, Wu Q, Zhao X, Wang X. The association between self-perceptions of aging and antihypertensive medication adherence in older Chinese adults. Aging Clin Exp Res. 2016;28(6):1113-20.

40. Robertson DA, Kenny RA. "I'm too old for that" - the association between negative perceptions of aging and disengagement in later life. Personal Individ Differ. 2016;100:114-9.

41. Levy BR, Ashman O, Slade MD. Age attributions and aging health: contrast between the United States and Japan. J Gerontol B Psychol Sc iSoc Sci. 2009;64(3):335-8.

42. Beyer AK, Wolff JK, Freiberger E, Wurm S. Are self-perceptions of ageing modifiable? Examination of an exercise programme with vs. without a selfperceptions of ageing intervention for older adults. Psychol Health. 2019; 34(6):661-76.

43. Liu X, Gu W, Li Z, Lei H, Li G, Huang W. Hypertension prevalence, awareness, treatment, control, and associated factors in Southwest China: an update. J Hypertens. 2017;35(3):637-44.

\section{Publisher's Note}

Springer Nature remains neutral with regard to jurisdictional claims in published maps and institutional affiliations. 